\title{
Plasma Brain-Derived-Neurotrophic Factor levels and cognitive function in euthymic bipolar type I patients
}

\author{
Vasco Videira Dias ${ }^{1,5^{*}}$, Sofia Brissos ${ }^{1,2}$, Benicio N Frey ${ }^{3}$, Ana Cristina Andreazza ${ }^{4}$, Flávio Kapczinski ${ }^{5}$, \\ Maria Luisa Figueira ${ }^{1}$ \\ From $1^{\text {st }}$ International Congress on Neurobiology and Clinical Psychopharmacology and European \\ Psychiatric Association Conference on Treatment Guidance \\ Thessaloniki, Greece. 19-22 November 2009
}

\section{Background}

Brain-derived neurotrophic factor (BDNF) is an important contributor to the pathophysiology of bipolar disorder (BD), and abnormalities in the BDNF-signaling system may be implicated in the cognitive decline observed in $\mathrm{BD}$ patients. We aimed to investigate serum BDNF levels in $\mathrm{BD}$ patients, and its relation with neurocognitive function.

\section{Materials and methods}

We measured serum BDNF levels using an enzymelinked immunosorbent assay method in 65 euthymic type I BD patients and 50 healthy controls, and administered a neuropsychological test battery to assess attention and mental control, perceptual-motor skills, executive functions, verbal fluency and abstraction, visuo-spatial attention, and memory.

\section{Results}

We found no significant differences regarding serum BDNF levels in BD patients and healthy controls. We found significant positive associations between serum BDNF levels and illness duration, and manic and depressive episodes in female BD patients only. Serum BDNF levels were lower in patients medicated with antipsychotics and/or lithium, whereas patients on valproate and/or antidepressants showed higher serum BDNF levels. Patients performed significantly worse on 11 out of 16 neurocognitive tests as compared to controls. We found a significant positive association between serum BDNF levels and a test of verbal fluency in both BD patients and controls.

${ }^{1}$ Bipolar Disorders Research Program, Hospital Santa Maria, Faculty of Medicine, University of Lisbon, (FMUL), Portugal

\section{Conclusions}

Present results support the hypothesis that BDNF normalizes with mood stabilization and pharmacological treatment. Our findings in young and physically healthy patients, with short illness duration and few mood episodes may explain the lack of association between serum BDNF levels and neurocognitive performance, even though cognitive performance in patients was overall significantly worse as compared to healthy controls.

\section{Author details}

'Bipolar Disorders Research Program, Hospital Santa Maria, Faculty of Medicine, University of Lisbon, (FMUL), Portugal. ${ }^{2}$ Centro Hospitalar Psiquiátrico de Lisboa, Lisbon, Portugal. ${ }^{3}$ Department of Psychiatry and Behavioral Neurosciences, McMaster University, Hamilton, ON, Canada. ${ }^{4}$ Department of Psychiatry, University of British Columbia, Vancouver, Canada. ${ }^{5}$ Bipolar Disorders Program and Laboratory of Molecular Psychiatry, Hospital de Clinicas de Porto Alegre, Brazil and INCT Translational Medicine.

Published: 22 April 2010

References

1. Hasler G, Drevets WC, Gould TD, Gottesman II, Manji HK: Toward Constructing an Endophenotype Strategy for Bipolar Disorders. Biol Psychiatry 2006, 60(2):93-105.

2. Rybakowski JK, Borkowska A, Skibinska M, Hauser J: Illness-specific association of val66met BDNF polymorphism with performance on Wisconsin Card Sorting Test in bipolar mood disorder. Mol Psychiatry 2006, 11(2):122-4.

3. Cunha ABM, Frey BN, Andreazza AC, et al: Serum brain-derived neurotrophic factor is decreased in bipolar disorder during depressive and manic episodes. Neurosc Lett 2006, 398:215-9.

4. Martinez-Aran A, Vieta E, Colom F, et al: Cognitive impairment in euthymic bipolar patients: implications for clinical and functional outcome. Bipolar Disord 2004, 6(3):224-32.

doi:10.1186/1744-859X-9-S1-S218

Cite this article as: Dias et al:: Plasma Brain-Derived-Neurotrophic Factor levels and cognitive function in euthymic bipolar type I patients. Annals of General Psychiatry 2010 9(Suppl 1):S218. 